\title{
A Comparative Study of Scheduling Disciplines in 5G Systems for Emergency Communications
}

\author{
Karina Gomez ${ }^{\dagger}$, Leonardo Goratti ${ }^{\dagger}$, Fabrizio Granelli ${ }^{\ddagger}$, and Tinku Rasheed ${ }^{\dagger}$ \\ ${ }^{\dagger}$ CREATE-NET, via alla Cascata 56D, 38123 Trento, Italy \\ ${ }^{\ddagger}$ DISI - University of Trento, Via Sommarive 14, 38100 Trento, Italy \\ Email: $\dagger\{$ name.surname $\} @$ create-net.org and łgranelli@disi.unitn.it;
}

\begin{abstract}
The hugely increasing demand for mobile broadband access to Web-based services and APPs will soon challenge even the UMTS Long Term Evolution (LTE) cellular technology and its advanced version LTE-A. For this reason the endeavor toward the Fifth Generation (5G) of cellular technology has started. Currently, there is no formal definition of 5G systems but they will encompass heterogeneity of radio technologies. System flexibility, (re)configuration and resilience will be indeed the catalysts of 5G. In this work, we delve different scheduling disciplines for communications of public safety users that are demanded to intervene during special events or in post disaster areas. In this extremely challenging environments we investigate which, out of several scheduling algorithms such as roundrobin, proportional fair and others, performs better. Since public safety users must be regarded with sufficient capacity as well as in a fair manner, we devise a novel scheduling algorithm for public safety compromising the mentioned performance metrics. Through Matlab simulations we will show the performance of the different algorithms even mixing commercial and public safety users. Our results show that the proposed scheduler performs well in the cases we studied, making it a good candidate for future 5G networks.
\end{abstract}

Keywords-Aerial-Terrestrial Networks; Emergency Communications; Public Safety; Resource Allocation; LTE Schedulers;

\section{INTRODUCTION}

The 4G cellular technology LTE and LTE-A is now approaching the mass market, promising an unprecedented quality of experience (QoE) for the users. Despite that, the pace at which mobile users consume broadband access to Webbased services and social networking forecasts a data traffic growth more than exponential. Therefore, the huge traffic volumes generated by mobile users is nowadays creating the need for the next generation of a full IP cellular technology, referred to as 5G [1]. Currently, there is no formal definition of $5 \mathrm{G}$ systems but requisites such as network flexibility, (re)configuration and resilience become even more crucial than ever. In this respect a paradigm shift is needed and great benefits are expected from applying network functions virtualization and software-defined networking approaches. Moreover, 5G systems could serve different types of customers including public safety (PS) users (or first responders) such as fire brigades and military forces. Indeed, the release 12 of 3GPP will enhance the features of LTE-Advanced to meet the requirements of PS communications [2]. Establishing common

The research leading to these results has received partial funding from the EC Seventh Framework Programme (FP7-2011-8) under the Grant Agreement FP7-ICT-318632. technical standards for commercial and PS networks offers several advantages to both communities.

This paper tackles the problem of efficient scheduling disciplines in future $5 \mathrm{G}$ communication systems where the requirements are those of PS users [2]. Since PS users are demanded to intervene in case of special events (e.g. world cup) or in post disaster areas, extremely high peaks of network capacity might be required. Furthermore, thinking that such a network could serve not only PS but also commercial users, challenges are even more exaggerated. 3GPP does not define specific uplink or downlink schedulers although several research proposals are available in the literature [3], [4]. Schedulers that are often implemented in LTE systems are best Channel Quality Indicator (BCQI), Proportionally Fair (PF) and Round Robin (RR) [5]. These schedulers target the best resources utilization for increasing fairness, throughput and bandwidth efficiency and assign them more promptly and faster to the users. Commercial and PS users typically have to satisfy different needs and requirements. The vital nature of PS communications clearly dictates radio coverage availability and minimum bit rate for all the users even in case of large concentration of people (typical of special events) and adversarial channel conditions (quite common in post disaster areas). To avoid dangerous loss of connectivity and consequent isolation of first responders located inside buildings or in tunnels it is mandatory to design smarter resource scheduling to relieve this severe problem.

In this paper, we delve a comparative study of different scheduling disciplines in 5G systems but mainly focusing on communications for PS users. On top of comparing different scheduling disciplines, we propose a simple yet effective uplink / downlink algorithm called PS scheduler that relies on the channel condition sub-grouping policies. The subgrouping policies classify the users based on their channel quality similar to the multicast channel scheduling used in [6]. The scheduler we propose here prioritizes PS users with poor channel conditions while trying to maximize the users' throughput. By means of Matlab simulations of the different schedulers we quantify the average cell throughput and fairness by comparing them. We further demonstrate that our PS scheduler is able to distribute more evenly the resources to the users, compromising with the overall system throughput.

The remaining part of the paper is organized as follows. Section 2 shows the related work. In Section 3, we present the system model while in Section 4 we describe the proposed scheduler. In Section 5, we detail the simulation work and the results obtained. Conclusions are provided in Section 6. 


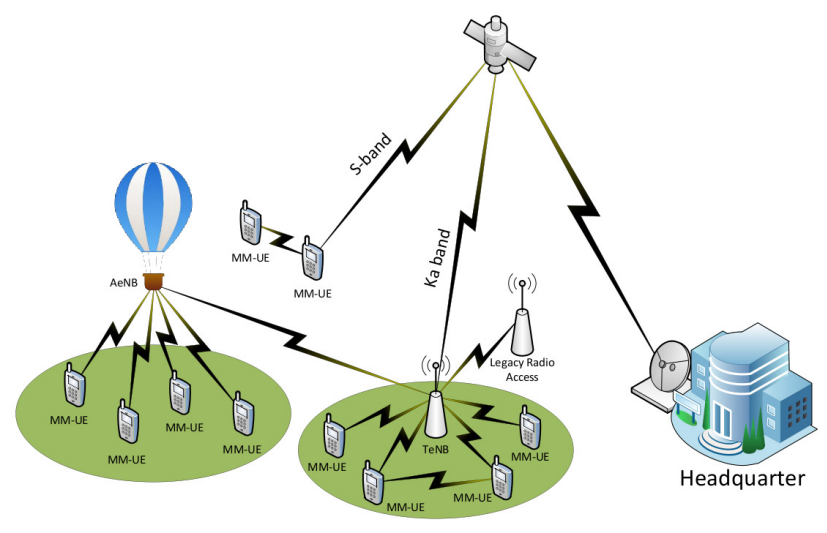

Fig. 1: ABSOLUTE Terrestrial-Aerial architecture.

\section{RELATED WORK}

The description we provide hereinafter is focused for simplicity on LTE technology, although it can be extended also to other systems. The scheduling of resources in LTE takes into account both radio and traffic conditions. In LTE, each physical channel (or resource block) has a corresponding quality indicator expressing channel conditions, different in uplink and downlink. In downlink, this information is provided by the user equipments (UEs) through the feedback of Channel Quality Indicators (CQIs). In uplink, the base station may use Sounding Reference Signals (SRSs) or other signals transmitted by the UEs to estimate the channel quality. A higher CQI value denotes a better channel condition with the CQIs given by 16 standard values [7].

\section{A. LTE-Based Commercial Networks}

The majority of the LTE-based scheduling schemes proposed in literature [4], [5] are based on maximizing fairness and throughput. In [4], authors design an LTE downlink scheduler by combining fast computing algorithms and resource scheduling. An exhaustive comparison of different schedulers is available in [5]. The BCQI scheduler is an unfair scheduling scheme in which only users with the best channel conditions are scheduled across the available RBs [8]. This scheduler aims to maximize the cell throughput but usually penalizes the users with worse channel conditions. To improve fairness the RR scheduler can be used since it is a fair, simple and easy scheme to implement [5]. In this case, users are scheduled with an equal amount of RBs without taking the CQI into account but penalizing the cell throughput while improving fairness.

A compromise between BCQI and RR is achieved by $\mathrm{PF}$ [3], [9]. This is based on a balancing strategy in which users are scheduled using a utility function that takes into account the CQI and the amount of RBs assigned. This scheduler tries to maximize the cell throughput while improving fairness at the same time. Similar to PF, the resource fair (RF) scheduler [10] tries to maximize the sum rate of all users while guaranteeing fairness with respect to the amount of RBs allocated to the users. The Maximum Minimum (MM) scheduler [10] achieves the best fairness maximizing the minimum of the user throughput, thus guaranteeing equal throughput to all the users. Notice that in MM scheduler the throughput of one user cannot be increased without decreasing the throughput of another user.

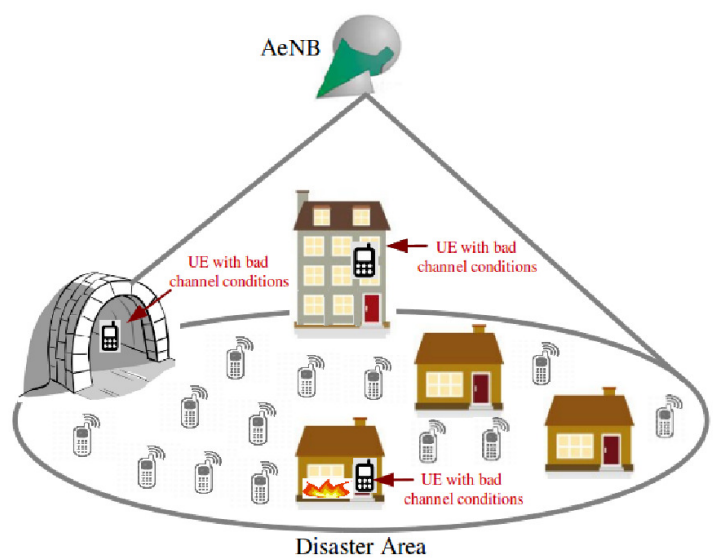

Fig. 2: Scenario considered for the LTE resource scheduling.

\section{B. LTE-Based Public Safety Networks}

The scheduler described above were designed for commercial networks and they are not optimized to catch all the peculiarities of communications in the field of public safety. Since LTE is the candidate technology for Ps communication systems, only few studies in the literature explicitly tackles scheduling in this emerging field. In [11] MAC level resources allocation for the purpose of uplink / downlink real-time video transmission is investigated. In this study, users are connected to Remote Radio Heads (RRHs) deployed over the cell and connected to a central base station. The authors argue that distributing RRHs in the cell improves the channel gains of all the users and reduces the negative effects of users with poor channel conditions. However, this consideration is not valid for disaster scenarios where part (or even all) the communication infrastructures could be destroyed.

\section{SYSTEM ARCHITECTURE}

The intervention of first responders can be greatly improved making available a communication system capable of offering broadband wireless access to interconnect different devices to local and remote control centers in a quick and reliable manner. In Fig. 1, the high-level representation of the architecture adopted in ABSOLUTE project ${ }^{1}$ (Hybrid Aerial-Terrestrial Architecture) is depicted. The architectural components are designed as standalone ground and aerial platforms that can be rapidly deployed in areas where physical access is impeded to first responders. Thus, low altitude platforms are deployed using helikites equipped with an LTE payload and capable of acting as base stations called Aerial eNBs (AeNB) [12]. Terrestrial Portable Land Mobile Units (PLMUs), equipped with an LTE interface, are also deployed to provide additional capacity and coverage for PS communications. Both AeNBs and PLMUs embed a satellite modem and antenna for connectivity to a GEO satellite station, enabling both inter-AeNBs and back-hauling services. Finally, Multi-Mode UEs equipped with LTE and satellite interfaces are used as handhelds. The role of each network component is to improve availability and reliability of the communications for the PS users.

In this paper, we analyze the scenarios (either during special events or after disasters) where some PS users experience

\footnotetext{
${ }^{1}$ ABSOLUTE EU FP7 Project, Available at: http://www.absolute-project.eu.
} 
TABLE I: Channel Quality Indicator.

\begin{tabular}{|c|c|c|c|}
\hline $\begin{array}{c}\text { CQI } \\
(\alpha)\end{array}$ & Modulation & $\begin{array}{c}\text { Approximate } \\
\text { Code Rate }\end{array}$ & $\begin{array}{c}\text { Information } \\
\text { bits per symbol }\end{array}$ \\
\hline \hline 0 & no transmission & - & - \\
1 & QPSK & 0.076 & 0.1523 \\
2 & QPSK & 0.120 & 0.2344 \\
3 & QPSK & 0.190 & 0.3770 \\
4 & QPSK & 0.300 & 0.6016 \\
5 & QPSK & 0.440 & 0.8770 \\
6 & QPSK & 0.590 & 1.1758 \\
7 & 16-QAM & 0.370 & 1.4766 \\
8 & 16-QAM & 0.480 & 1.9141 \\
9 & 16-QAM & 0.600 & 2.4063 \\
10 & 64-QAM & 0.450 & 2.7305 \\
11 & 64-QAM & 0.550 & 3.3223 \\
12 & 64-QAM & 0.650 & 3.9023 \\
13 & 64-QAM & 0.750 & 4.5234 \\
14 & 64-QAM & 0.850 & 5.1152 \\
15 & 64-QAM & 0.930 & 5.5547 \\
\hline
\end{tabular}

poor channel conditions. Fig. 2 shows the scenario that we deem to investigate for the schedulers. In this scenario, the communication of public safety users inside buildings, tunnels or even building on fire are affected by bad channel conditions. Therefore, the scheduling is done at the MAC layer and users with bad channels conditions are scheduled first. A critical aspect that we need to take into account is that the channel state information available at the AeNB side for the UEs must be up-to-date. In this work, we assume that the CQI value of each RB on a per user basis is available at the AeNB in each Transmission Time Interval (TTI).

\section{Scheduler for Public Safety Communications}

We consider an LTE-based cellular network where one base station is serving a set of $m$ UEs, each UE is denoted by ith $(i=1,2, \ldots, m)$. The downlink bandwidth is divided into a set of maximum $n \mathrm{RBs}$, each $\mathrm{RB}$ is denoted by $j$ th $(j=1,2, \ldots, n)$, which will be inputs of the scheduling vector $\Delta(1,2, \ldots, n)$. We assume that the base station has always at least one packet awaiting to be transmitted to each UE (traffic saturation conditions). In terms of scheduled resources, a UE can be assigned with a minimum of one RB in frequency and one TTI (simple denoted by $t$ hereinafter) over time. We now define the matrix $\boldsymbol{\Gamma}$, whose values $\alpha_{i j}$ denote the CQI corresponding to UE $i$ and RB $j$. In the base station each $\alpha_{i j}$ can be represented by 16 standard values as specified in [7] and shown in Table I. At time $t$, we compute the average CQI $\left(\bar{\alpha}_{i}\right)$ for the $i$ th UE, where we take the values in $\Gamma$ and we average with respect to the $j$ RBs. After that, the scheduling priorities of the UEs in the proposed scheduler is done organizing the $\bar{\alpha}_{i}$ in descending order. The lower the average CQI value, the higher the scheduling priority. The average CQI value is computed as follows

$$
\bar{\alpha}_{i}(t)=\frac{1}{n} \cdot\left(\sum_{j=1}^{n} \alpha_{i j}(t)\right) .
$$

Each value of $\alpha$ corresponds to a specific modulation and coding scheme (MCS), determining in this way the maximum capacity of a RB. Therefore, the information carried by the RBs depends on $\alpha_{i j}$. The value of $\alpha$ maximizing the capacity

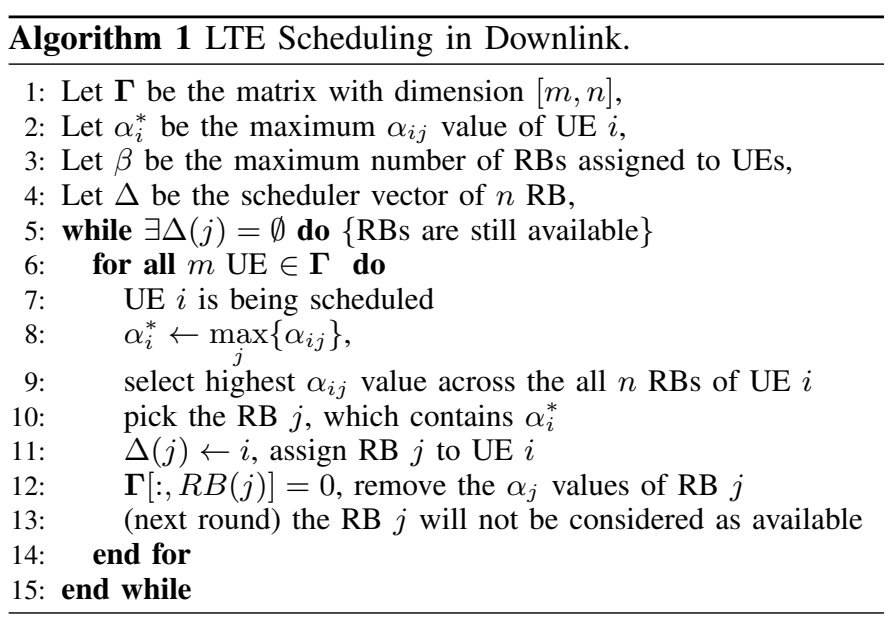

of the UE $i$ per RB $j$ in each time $t$ is the following

$$
\alpha_{i}^{*}(t)=\max _{j}\left\{\alpha_{i j}(t)\right\},
$$

where $\alpha_{i}^{*}$ is the best available RB for each UE $i$ at each $t$. To distribute equally the $n$ RBs to the set of $m$ UEs, the maximum number of RBs assigned to UE $i$ is denoted by $\beta$ and calculated as follows

$$
\beta(t)=\left\{\begin{array}{ll}
1 & \text { if } \frac{n}{m} \leq 1 \\
\left\lfloor\frac{n}{m}\right\rfloor & \text { if } \frac{n}{m}>1
\end{array} .\right.
$$

\section{A. LTE Scheduling in Downlink}

The PS scheduler makes use of equations (2) and (3) for scheduling the UEs in the best available RBs as illustrated in Algorithm 1. The proposed PS algorithm schedules the UEs as described in the steps below.

- Step 1: UEs in $\Gamma$ are scheduled with a priority decreasing as $\bar{\alpha}_{i}$ increases. In this way, the UEs with poor channel conditions are scheduled first using the best available RBs in terms of CQI (see line 5 in Algorithm 1).

- $\quad$ Step 2: For each UE in $\boldsymbol{\Gamma}$, the highest $\alpha_{i j}$ value across the $n$ available RBs is selected $\left(\alpha_{i}^{*}\right)$. Thus, the best RB $j$ for allocating the UE is identified (see lines 7, 8 and 9 in Algorithm 1).

- Step 3: The RB $j$ is allocated to UE $i$ and marked in the scheduling vector $\Delta$ (see lines 10 and 11 in Algorithm 1). Then the RB $j$ is deleted from the set of available RBs and it will not be available at the next iteration (see line 12 in Algorithm 1).

The PS scheduler iterates steps $\mathbf{2}$ and $\mathbf{3}$ until all RBs have been assigned to the UEs (see line 5 in Algorithm 1).

\section{B. LTE Scheduling in Uplink}

The PS uplink scheduler takes into account the constraint that in LTE only RBs that are consecutive in frequency can be allocated to the same UE. Thus, the RBs are divided in groups of $\beta \mathrm{RBs}$ each (a group of RBs is indexed with $k$ ). The total number of non-overlapping groups that can be created with $n$ RBs is equal to the integer part of $n / \beta$. The $\alpha_{i j}$ values $^{2}$ of

\footnotetext{
${ }^{2}$ Notice that we assume that CQIs may be also used as indicators for uplink channel conditions, since using SRSs or CQIs will not affect the general purpose of the proposed uplink PS.
} 


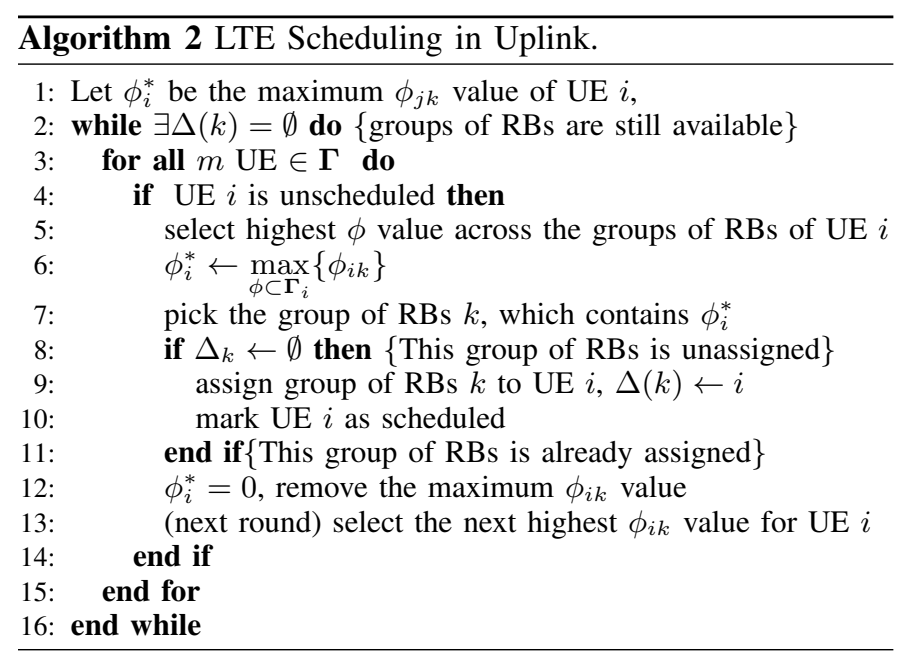

each group of RBs are aggregated in the variable $\phi_{i k}$ as follow $\phi_{i k}=\left[\alpha_{i j}(t)+\alpha_{i(j+1)}(t)+\ldots+\alpha_{i(j+\beta)}(t)\right]$. The value of $\phi$ maximizing the capacity of UE $i$ in each time $t$ is the following

$$
\phi_{i}^{*}(t)=\max _{k}\left\{\phi_{i k}(t)\right\}
$$

where $\phi_{i}^{*}$ is the best available group of RBs for each UE $i$ at each $t$. The PS scheduler relies on equation (4) for scheduling the UEs across the group of best available RBs, as described in Algorithm 2 and summarized below.

- Step 1: As before, the scheduling priority of the UEs in $\Gamma$ is organized with respect to $\bar{\alpha}_{i}$ and in particular assigning a higher priority to UEs with lower $\bar{\alpha}_{i}$. UEs with poor channel conditions are scheduled first (see line 3 in Algorithm 2).

- $\quad$ Step 2: For each (unscheduled) UE in $\boldsymbol{\Gamma}$, the highest $\phi_{i}$ value across the available group of RBs is selected $\left(\phi_{i}^{*}\right)$. Thus, the best group of RBs for allocating the $\mathrm{UE}$ is identified (see lines 5, 6 and 7 in Algorithm 2).

- $\quad$ Step 3: If the group of RBs $k$ is unassigned, this group is allocated to UE $i$ in the scheduling vector $\Delta$ and the UE $i$ is marked as scheduled (see lines 9 and 10 in Algorithm 2).

- $\quad$ Step 4: If the group of $\mathrm{RBs} k$ is already assigned, this group is deleted from the set of available RBs. Thus, the next group of RBs with the highest $\phi_{i k}$ will be selected at the next iteration (see lines 12 and 13 in Algorithm 2).

The PS scheduler iterates steps 2, 3 and $\mathbf{4}$ until all the groups of RBs have been assigned to the UEs.

Fig. 3 shows an illustrative example of downlink and uplink scheduling of UEs with bad and good channel conditions, respectively. For clarity, the example is limited to a set of 3 UEs camping in a single cell with 6 available RBs. The green colored cells highlight the RBs selected following the policies of a-b) BCQI, c-d) RR and f-g) PS schedulers. The proposed PS scheduler provides a compromise in distributing the available RBs among UEs with good and bad channel conditions.

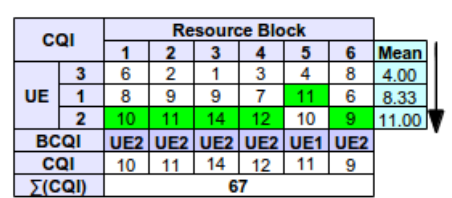

(a) Downlink BCQI Scheduler

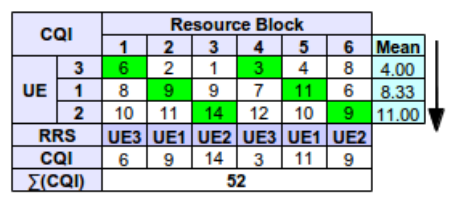

(c) Downlink RR Scheduler

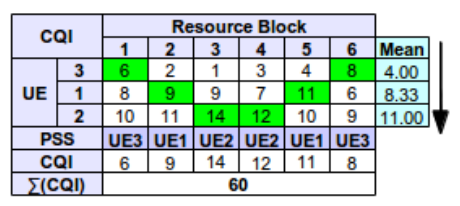

(e) Downlink PS Scheduler

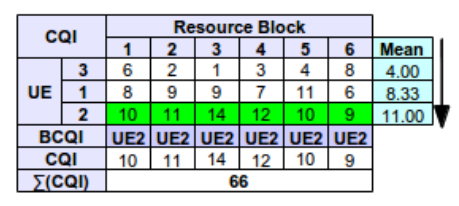

(b) Uplink BCQI Scheduler

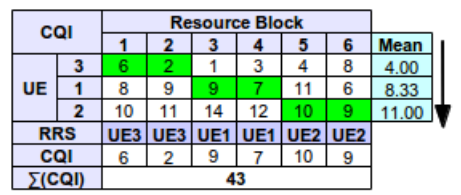

(d) Uplink RR Scheduler
Fig. 3: Example for schedulers using different policies.

\section{PERFORMANCE EVALUATION}

To compare the different schedulers, a MATLAB-based LTE simulator is used [13]. The comparison is done in terms of achieved throughput and fairness with MM, RR, PF, RF and BCQI schedulers [10]. The fairness is quantified using Jains fairness index [14], denoted by $\mathcal{J}$, which measure the fairness among the users. The index $\mathcal{J}$ is a set of values for the $m$ users as shown below

$$
\mathcal{J}\left(T_{1}, T_{2}, \ldots, T_{m}\right)=\frac{\left(\sum_{i=1}^{m} T_{i}\right)^{2}}{m \cdot \sum_{i=1}^{m} T_{i}^{2}},
$$

where $T_{i}$ is the throughput for the $i t h$ user. The ideal case of fairness is achieved when the index $\mathcal{J}$ is equal to 1 reflecting the best case (all users receive equal resources). While decreasing fairness is reflected with decreasing value of the $\mathcal{J}$ index.

Table II shows the simulation parameters used in the LTE simulator based on the 3GPP specifications [7]. We focus on the AeNB network and we consider that the commercial infrastructure could be destroyed or overloaded. The simulation setup consists of a single cell, where one AeNB with an omnidirectional antenna is located at the center (coverage radius equal to $700 \mathrm{~m}$ ) and SISO discipline is applied. The cell is configured in Frequency-Division Duplex mode in uplink and downlink. Bandwidth values used are 1.4, 3, 5, 10, 15 and $20 \mathrm{MHz}$ in both downlink and uplink considering QPSK, 16-QAM and 64-QAM modulations. These bandwidths are equivalent to $6,15,25,50,75$, and $100 \mathrm{RBs}$ respectively. In the simulation a variable number of UEs is assumed, [5, $10,20,30,40,50]$, which are uniformly distributed inside the cell. An 3GPP typical urban channel model with a NLOS communication path is simulated [7]. We further assume that the average receive signal-to-noise-ratio (SNR) is distributed in the range of i) $2 \mathrm{~dB}$ to $4 \mathrm{~dB}$ for the $50 \%$ of the users, and ii) $18 \mathrm{~dB}$ to $36 \mathrm{~dB}$ for the rest of the users. To map the channel conditions of the users, CQI values are generated as specified in [13]. Traffic is modeled with a infinite backlog of packets 
TABLE II: Simulation parameters and assumptions [7].

\begin{tabular}{r|l} 
Parameter & Value \\
\hline \hline Duplex Mode & Frequency-Division \\
System bandwidths (UL/DL) & {$[1.4,3,5,10,15$ 20] MHz } \\
Number of RBs & {$[6,15,25,50,75,100]$} \\
Modulation & QPSK, 16-QAM, 64-QAM \\
User distribution & Uniform \\
Traffic model & Infinitely backlogged \\
Channel Model & 3GPP Typical Urban \\
Served users & {$[5,10,20,30,40,50]$} \\
Antenna configuration & 1 transmit, 1 receive (1 x 1) \\
Receiver & Zero Forcing ZF \\
Schedulers & BCQI, PF, RF, MM, RR, PS \\
\hline
\end{tabular}

in which nodes are in saturation conditions. The simulation results are averaged over 1000 different simulations (notice that the $95 \%$ confidence interval is too small to be identified in the figures).

Figure 4 shows the performance of different schedulers in terms of cell throughput and fairness versus LTE bandwidth. The cell is serving 20 users (50\% of which experience an average SNRs lower than $4 \mathrm{~dB}$ ). Figures are provided for BCQI, PF, RF, RR, MM and PS schedulers for the bandwidths equal to $1.4,3,5,10,15$ and $20 \mathrm{MHz}$, respectively. Notice that for the 1.4 and $3 \mathrm{MHz}$ bandwidths, which corresponds to 6 and 15 RBs respectively, the number of resources are lower than the number of users $(\beta<1)$. Figure 4.a shows that for all LTE bandwidths the highest cell throughput is achieved using the BCQI scheduler since it serves only users with good channel conditions. The lowest cell throughput is instead achieved using the RR scheduler since it allocates the resources without taking into account the channel conditions of the users. Similar cell throughput is achieved using the MM scheduler since it maximizes the minimum of the users' throughput. A compromise is achieved using PF and RF schedulers since the channel conditions of the users are taken into account for allocating the resources. Finally, the cell throughput achieved using PS scheduler is lower than PF and RF since the scheduler prioritizes the users with bad channel condition. However, PS scheduler performs better than RR and MM schedulers for the LTE bandwidths equal to $5,10,15$ and $20 \mathrm{MHz}$, in which the number of resources are larger than the number of users $(\beta>1)$. Focusing on fairness, the best is achieved with the MM scheduler since it divides the resources equally amongst the users, whereas the worst fairness is achieved with the BCQI scheduler as expected. The RR scheduler performs better than BCQI but worse than PF, RF and PS. Out of this three, PS scheduler has better fairness than PF and RF when the system has not allocated all resources, which is good for the users with bad channel conditions. Good fairness is also achieved with the $\mathrm{PF}$ and RF schedulers complementing the good performance achieved in terms of throughput.

Figure 5 shows the performance of different schedulers in terms of cell throughput and fairness versus the number of served users. The cell is configured with $10 \mathrm{MHz}$ bandwidth (50\% of the users experience an average SNR lower than $4 \mathrm{~dB}$ for all cases). We can observe that similar results in terms of cell throughput and fairness are achieved as the number of users increases. Observations done before for the different schedulers are also valid in case of Figure 5. Notice that the results of fairness and cell throughput prove the

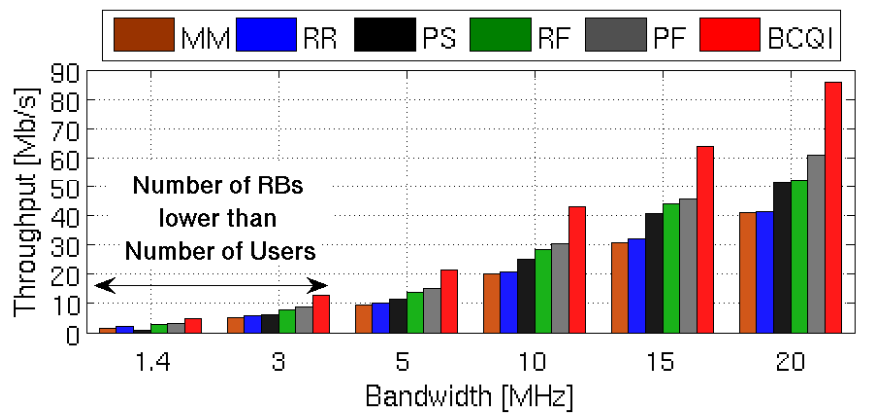

(a) Cell throughput achieved with different schedulers.

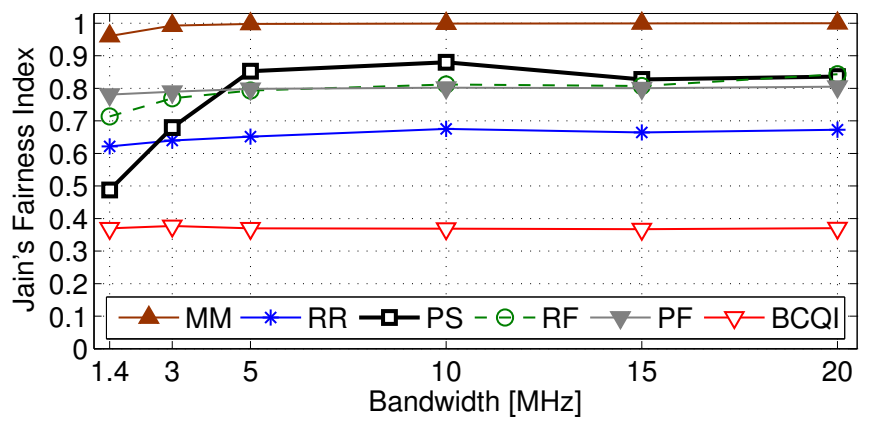

(b) Fairness achieved with different schedulers.

Fig. 4: Performance of different schedulers versus LTE bandwidth. The cell is made of 20 users $(50 \%$ of the users have average SNRs lower than $4 \mathrm{~dB}$ ).

effectiveness of the proposed PS scheduler for distributing the available resources amongst the users, though at the cost of lowering the cell throughput. This is due tot he fact that the PS scheduler prioritizes the users with poor channel conditions during resources assignment. Based on the results we obtained, we remark the following:

- $\quad B C Q I$ is not recommended for emergency communications. The scheduling excludes users with poor channel conditions, which is optimal for commercial networks but not for emergency communications in which fairness is crucial.

- $\quad M M$ is also not recommended for emergency communications where severe propagation conditions of the radio signal are challenging. In these cases, the MM scheduler penalizes the users with good channel conditions in terms of throughput since the goal is to maximize the minimum of the users' throughput.

- $R R$ scheduler does not take into account channel conditions for resources allocation (not optimal for taking advantages of $5 \mathrm{G}$ system features). It penalizes throughput and fairness. The main advantage of RR scheduler is simplicity in assigning the available resources to the users.

- $\quad P F$ and $R F$ maintain a good balance between the objective of maximizing cell throughput and fairness. These schedulers are a good compromise in both emergency and commercial communications.

- $\quad P S$ provides the best trade-off in terms of cell throughput and fairness in all cases of several users with 


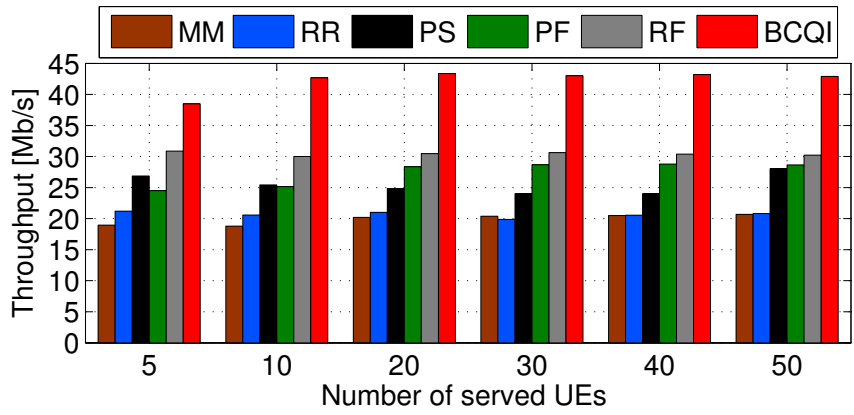

(a) Cell throughput achieved with different schedulers.

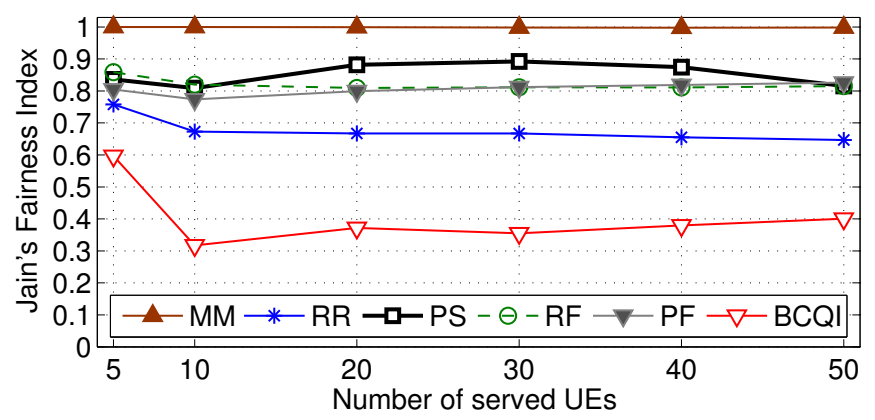

(b) Fairness achieved with different schedulers.

Fig. 5: Performance of different schedulers versus number of served users in a cell configured with $10 \mathrm{MHz}$ bandwidth (50\% of the users experience an average SNR lower than $4 \mathrm{~dB}$ ).

bad channel conditions. Another advantage of PS scheduler is a reduced implementation complexity and faster assignment of the resources specially compared to PF and RF.

It is worth pointing out that the combinations of PS and PF schedulers can be a good option for 5G systems serving both PS and commercial users. In the following example, we simulate an LTE cell configured with $10 \mathrm{MHz}$ bandwidth, when 40 users are uniformly distributed within the cell coverage. Out of the total users, 20 are first responders (10 users experience an average SNRs lower than $4 \mathrm{~dB}$ ) and other 20 are commercial users. We perform simulations for PS and PF individually as well as combining them. The combined scheduler (CS) is created using the $50 \%$ of the RBs for scheduling the PS users according to PS discipline and the other $50 \%$ of the RBs for scheduling the commercial users according to the PF discipline. Figure 6 shows the performance of PF, PS and CS. As it can be seen, the figures highlight that the combinations of PS and PF schedulers can be beneficial for both cell throughout and fairness.

\section{Vi. CONCLUSions}

In this paper, we provided a comparative study of different scheduling disciplines that can be used in future $5 \mathrm{G}$ systems but focusing on the increasingly important field of emergency communications. We also proposed and studied the performance of a simple yet effective uplink / downlink PS scheduler for LTE-based emergency communications. The proposed scheduler uses the channel condition sub-grouping policies for scheduling users with bad channel conditions. The proposed scheduler assigns higher scheduling priority to

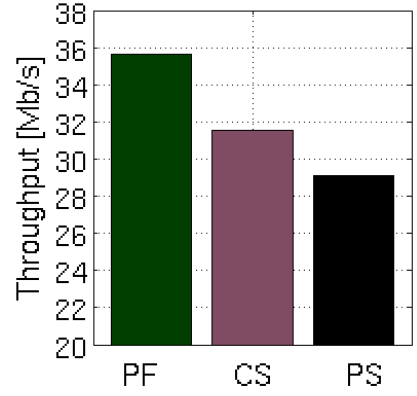

(a) Cell throughput

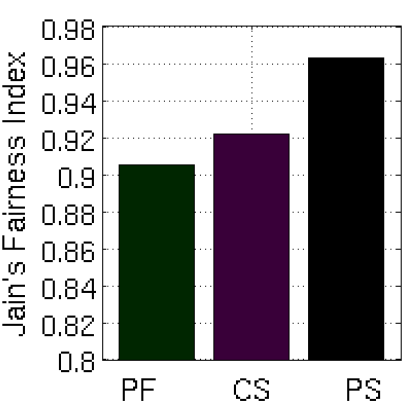

(b) Fairness
Fig. 6: Performance of different schedulers in a cell configured with $10 \mathrm{MHz}$ bandwidth.

users with poor channel conditions while trying to achieve a cell throughput as high as possible. Simulation results show the effectiveness of the different scheduling disciplines by comparing them in terms of cell throughput and fairness. Therefore, we are entitled to conclude that the proposed PS scheduler well compromises throughput and fairness. We also proved that the combinations of PS and PF schedulers can be beneficial for both cell throughout and fairness when serving public safety and commercial users at the same time.

\section{REFERENCES}

[1] G. Fettweis and S. Alamouti, "5G: Personal mobile internet beyond what cellular did to telephony," Communications Magazine, IEEE, vol. 52, no. 2, pp. 140-145, February 2014.

[2] T. Doumi, M. Dolan, S. Tatesh, A. Casati, G. Tsirtsis, K. Anchan, and D. Flore, "LTE for public safety networks," IEEE Communication Magazine, vol. 51, no. 2, 2013.

[3] L. Ruiz de Temino, G. Berardinelli, S. Frattasi, and P. Mogensen, "Channel-aware scheduling algorithms for SC-FDMA in LTE uplink," in IEEE PIMRC, Sept 2008, pp. 1-6.

[4] B. Sadiq, R. Madan, and A. Sampath, "Downlink scheduling for multiclass traffic in LTE," EURASIP J. Wireless Communication Networks, vol. 2009, pp. 14:9-14:9, Mar. 2009.

[5] O. Osterbo, "Scheduling and capacity estimation in LTE," in IEEE ITC, 2011, pp. 63-70.

[6] G. Araniti, M. Condoluci, L. Militano, and A. Iera, "Adaptive resource allocation to multicast services in LTE systems," IEEE Transaction on Broadcasting, vol. 59, no. 4, pp. 658-664, 2013.

[7] "Technical Specification Group Radio Access Networks; Deployment aspects (Release 8)," Available at: http://www.3gpp.org/ftp/Specs/htmlinfo/25943.htm.

[8] S. Schwarz, C. Mehlfuhrer, and M. Rupp, "Throughput maximizing multiuser scheduling with adjustable fairness," in IEEE ICC, 2011.

[9] R. Kwan, C. Leung, and J. Zhang, "Proportional fair multiuser scheduling in LTE," IEEE SPL, vol. 16, no. 6, 2009.

[10] S. Schwarz, C. Mehlfuhrer, and M. Rupp, "Low complexity approximate maximum throughput scheduling for LTE," in IEEE ASILOMAR, Nov 2010.

[11] E. Yaacoub and O. Kubbar, "On the performance of distributed base stations in LTE public safety networks," in IEEE IWCMC, Aug 2012.

[12] L. Reynaud, R. Hermenier, I. Bucaille, K. Sithamparanathan, M. Mohorcic, M. Smolnikar, A. Munari, K. Gomez, T. Rasheed, and A. Valcarce, "Airborne base stations for emergency and temporary events," PSATS, June 2013.

[13] J. C. Ikuno, M. Wrulich, and M. Rupp, "System level simulation of LTE networks," in IEEE VTC, Taipei, Taiwan, May 2010.

[14] R. Jain, D.-M. W. Chiu, and W. R. Hawe, "A Quantitative Measure Of Fairness And Discrimination For Resource Allocation In Shared Computer Systems," Digital Equipment Corp., Tech. Rep., Sep. 1984. 